\title{
Methamphetamine Learning Induces Persistent and Selective Nonmuscle Myosin II-Dependent Spine Motility in the Basolateral Amygdala
}

\author{
Erica J. Young, ${ }^{1,2}$ Hua Lin, ${ }^{1}$ Theodore M. Kamenecka, ${ }^{1}$ Gavin Rumbaugh, ${ }^{2}$ and Courtney A. Miller ${ }^{1,2}$ \\ Departments of ${ }^{1}$ Molecular Medicine, and ${ }^{2}$ Neuroscience, The Scripps Research Institute, Jupiter, Florida 33458
}

Nonmuscle myosin II inhibition (NMIIi) in the basolateral amygdala (BLA), but not dorsal hippocampus (CA1), selectively disrupts memories associated with methamphetamine $(\mathrm{METH})$ days after learning, without retrieval. However, the molecular mechanisms underlying this selective vulnerability remain poorly understood. A known function of NMII is to transiently activate synaptic actin dynamics with learning. Therefore, we hypothesized that METH-associated learning perpetuates NMII-driven actin dynamics in synapses, leading to an extended window of vulnerability for memory disruption. We used time-lapse two-photon imaging of dendritic spine motility in acutely prepared brain slices from female and male mice following METH-associated learning as a readout of actin-myosin dynamics. Spine motility was persistently increased in the BLA, but not in CA1. Consistent with the memory disrupting effect of intra-BLA NMII inhibition, METH-induced changes to BLA spine dynamics were reversed by a single systemic injection of an NMII inhibitor. Intra-CA1 NMII inhibition, on the other hand, did not disrupt METH-associated memory. Thus, we report identification of a previously unknown ability for spine actin dynamics to persist days after stimulation and that this is under the control of NMII. Further, these perpetual NMII-driven spine actin dynamics in BLA neurons may contribute to the unique susceptibility of METH-associated memories.

Key words: actin; addiction; cytoskeleton; dendrititic spine; drug seeking; hippocampus

Significance Statement

There are no Food and Drug Administration-approved pharmacotherapies to prevent relapse to the use of stimulants, such as methamphetamine (METH). Environmental cues become associated with drug use, such that the memories can elicit strong motivation to seek the drug during abstinence. We previously reported that the storage of METH-associated memories is uniquely vulnerable to immediate, retrieval-independent, and lasting disruption by direct actin depolymerization or by inhibiting the actin driver nonmuscle myosin II (NMII) in the BLA or systemically. Here we report a potential structural mechanism responsible for the unique vulnerability of METH-associated memories and METH-seeking behavior to NMII inhibition within the BLA.

\section{Introduction}

Dendritic spines, actin-rich postsynaptic compartments, are dynamic and enable input-specific biochemical and electrical isolation of synapses to facilitate information storage (Kasai et al., 2003; Araya et al., 2006a; Dumitriu et al., 2007). During learning, spines undergo structural and functional changes to stabilize synapses and, ultimately, memory (Lai et al., 2012; Hayashi-Takagi

\footnotetext{
Received Sept. 10, 2019; revised Jan. 17, 2020; accepted Feb. 4, 2020.

Author contributions: E.J.Y., G.R., and C.A.M. designed research; E.J.Y. performed research; H.L. and T.M.K. contributed unpublished reagents/analytic tools; E.J.Y., G.R., and C.A.M. analyzed data; C.A.M. wrote the paper.

This work was funded by National Institute on Drug Abuse Grants DA-034116 and DA-049544 (C.A.M.). We thank all members of the Miller and Rumbaugh Laboratories for technical assistance and thoughtful discussions. The authors declare no competing financial interests.

Correspondence should be addressed to Gavin Rumbaugh at gavin@scripps.edu or Courtney A. Miller at cmiller@scripps.edu.

https://doi.org/10.1523/JNEUROSCI.2182-19.2020

Copyright $\odot 2020$ the authors
}

et al., 2015). Consistent with this, there is a tight connection between the physical geometry of spines and the ability to transform experiences into long-term memory (Xu et al., 2009; Yang et al., 2009; Makino and Malinow, 2011). Actin polymerization, the elongation and complex branching of filamentous actin, drives the spine structural plasticity that is required for functional plasticity and learning (Kim and Lisman, 1999; Smart and Halpain, 2000; Matsuzaki et al., 2001; Star et al., 2002; Noguchi et al., 2005, 2011).

Long-term potentiation (LTP) and newly formed memories become impervious to actin depolymerization shortly after the underlying synaptic plasticity occurs (Mantzur et al., 2009; Rehberg et al., 2010; Rex et al., 2010). This is attributed to rapid stabilization of the actin cytoskeleton through the cessation of polymerization and recruitment of actin capping and stabilizing proteins (Lamprecht, 2014). However, we recently discov- 
ered that memories associated with the commonly abused stimulant, methamphetamine $(\mathrm{METH})$, remain uniquely susceptible to depolymerization days after learning (Young et al., 2014). Indeed, a single infusion of the actin depolymerizer Latrunculin A (LatA) into the basolateral amygdala (BLA) results in an immediate, long-lasting, and retrieval-independent loss of METH-associated memory and associated drug-seeking behavior. This memory loss is accompanied by a return of BLA spine density to pre-METH conditioning levels. LatA influences populations of dynamic, but not stable, actin by sequestering monomers (Morton et al., 2000; Yarmola et al., 2000). Thus, the susceptibility of a METH-associated memory days after learning suggests that METH may interfere with actin stabilization in BLA spines.

The motivation of drug-associated memories is triggered, in large part, by activation of the amygdala (Childress et al., 1999; Miller and Marshall, 2005; Otis et al., 2015), making the ability to rapidly and selectively disrupt amygdala-dependent METH-associated memories a potential therapeutic avenue (Young et al., 2015). Because the critical role of actin in numerous cellular processes limits its therapeutic potential, we shifted focus to the molecular motor ATPase, nonmuscle myosin II (NMII), a driver of actin polymerization (Ryu et al., 2006; Koskinen et al., 2014; Omotade et al., 2017). NMII is a hexameric protein made of two heavy chains (MHC), two regulatory light chains (RLCs), and two essential light chains. The MHCs are the workhorse, bearing both the ATPase and actin-binding sites, and motor heads that bind actin and move it through physical force. We have demonstrated that NMII drives synaptic actin polymerization to promote and stabilize LTP and long-term memory formation in CA1 and the BLA (Rex et al., 2010; Gavin et al., 2012). In addition, through genetic and pharmacologic manipulations, we have established that targeting NMII in the BLA or systemically is well tolerated and recapitulates the METH-associated memory, drug-seeking, and spine density effects of direct actin depolymerization (Young et al., 2016, 2017).

The rapid and persistent impact of NMII inhibition on METH-associated memory is surprisingly specific, having no such effect on memories associated with footshock, food reward, or other drugs of abuse (nicotine, morphine, cocaine, or mephedrone; Briggs et al., 2017). The underlying mechanism responsible for this selectivity is largely
A
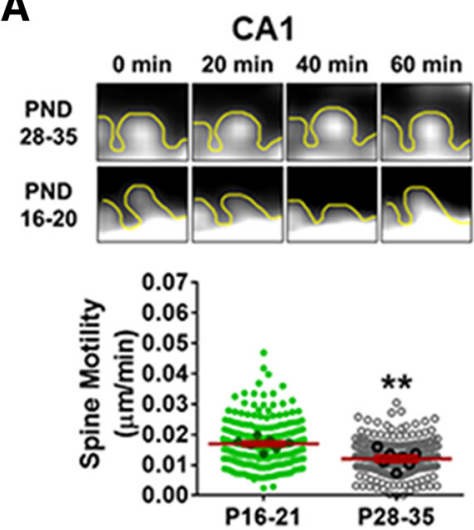

B

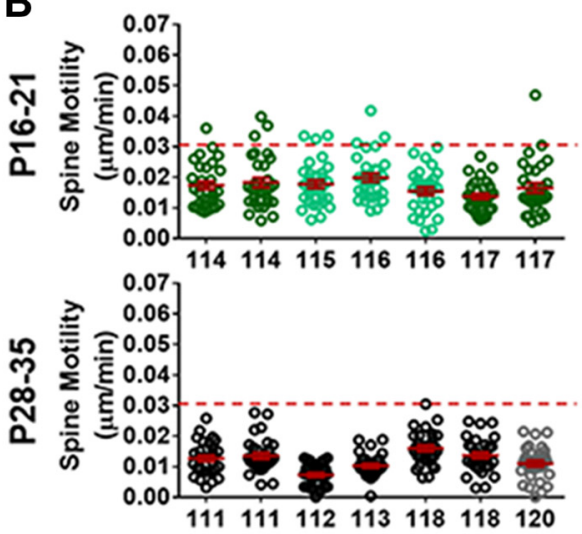

C

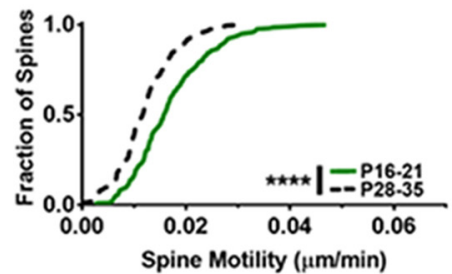

D
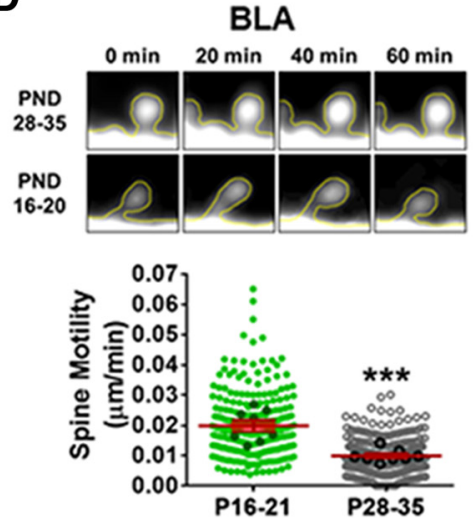

E

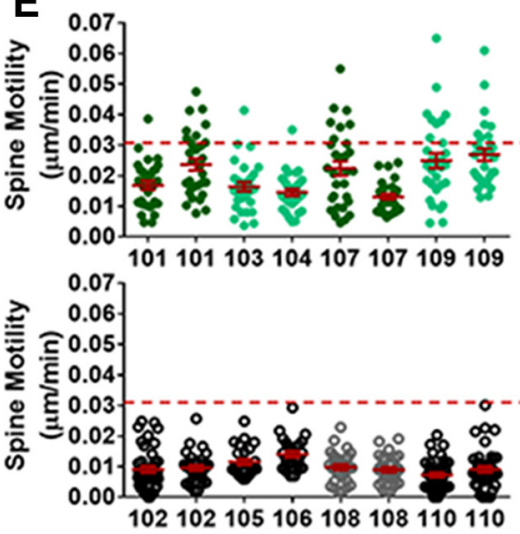

$\mathbf{F}$

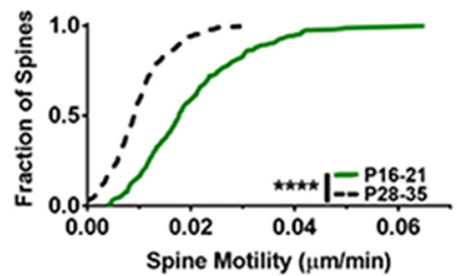

G

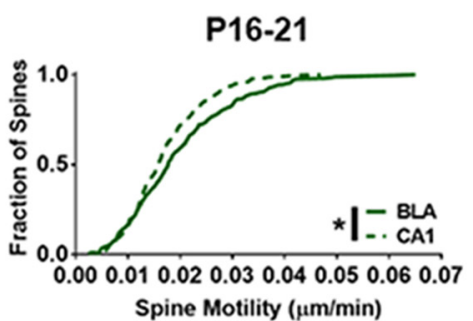

Between Region Comparisons

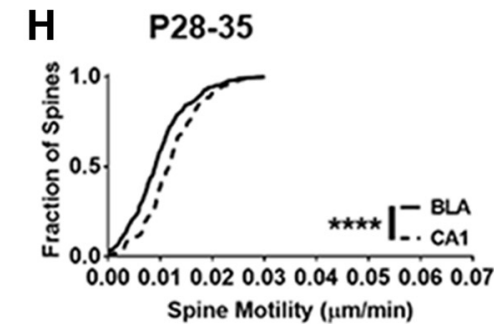

Figure 1. Motility of $C A 1$ and BLA spines decreases with age. $A, C A 1$ dendritic spine motility from naive young animals (P16P21) and early adolescent animals (P28 -P35). Small lighter colored circles represent the movement of each individual spine over $1 \mathrm{~h}$, while the large darker circles represent the average of 30 spines from each slice (P28 -P35: $n=210$ spines, 7 slices, 5 animals; P6-P21: $n=210$ spines, 7 slices, 4 animals). $\boldsymbol{B}$, CA1 spine motility organized by animal and slice. Slices from female animals are in light green and gray, males are depicted by dark green and black. $C$, Cumulative distributions of CA1 spine movements from P16 -P21 and P28 -P35. D-F, Spine movements measured in BLA slices from P16 -P21 and P28 -P35 (D, E), and corresponding cumulative distributions ( $\boldsymbol{F} ; \mathrm{P} 28-\mathrm{P} 35: n=260$ spines, 8 slices, 5 animals; P16-P21: $n=240$ spines, 8 slices, 5 animals). $\mathbf{G}, \boldsymbol{H}$, Comparison of BLA and CA1 spine movements at P16-P21 and P28-P35. ${ }^{*} p<0.05,{ }^{* *} p<0.01,{ }^{* * *} p<0.0001$.

unknown to date. The impact of actin depolymerization on BLA spine density via LatA or NMII inhibition with blebbistatin (Blebb) indicates a connection to spine actin. Therefore, we hypothesized that the selective susceptibility of METH-associated 
A

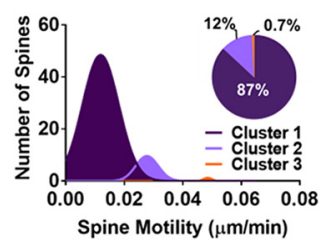

B
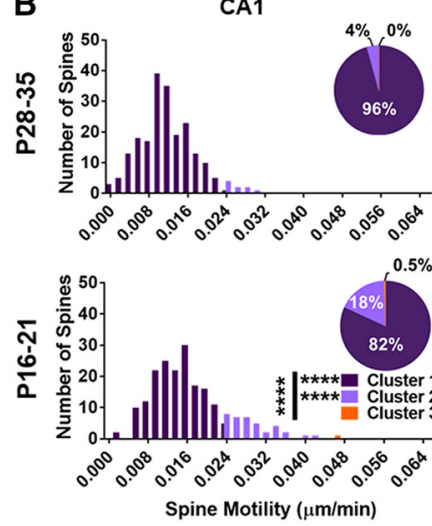

C
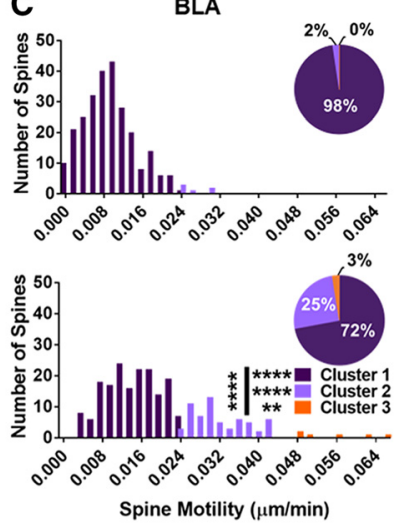

Figure 2. Cluster analysis of $C A 1$ and $B L A$ age-related spine movements. $A, A l l C A 1$ and BLA spine movements underwent cluster analysis. $\boldsymbol{B}, \boldsymbol{C}$, Distribution of spine movements within each group across three clusters. Error bars represent SEM. ${ }^{* *} p<0.01,{ }^{* * *} p<0.001,{ }^{* * *} p<$ 0.0001 .

memory is due to NMII-dependent sustained spine actin dynamics. As a first step toward addressing this, we used time-lapse two-photon imaging of actin-dependent BLA spine motility, as measured by the dynamic extension and retraction of the spine head from the dendrite. CA1 spine motility was measured for comparison. Like the BLA, CA1 is a critical component of the neural circuit supporting drug-associated memories, and we have reported spine density increases in CA1 with METH learning, as in the BLA (Kutlu and Gould, 2016; Briggs et al., 2017). However, METH memories do not have the same vulnerability to NMII disruption in CA1. And, unlike BLA spines, METHinduced CA1 spine density increases remain intact after systemic Blebb treatment (Briggs et al., 2017). Therefore, we anticipated that METH conditioning would result in a NMII-dependent increase in spine motility in the BLA, but not in CA1.

\section{Materials and Methods}

All animal procedures were conducted in accordance with the Scripps Research Animal Care and Use Committee and national regulations and policies.

\section{Animals}

Male and female heterozygous Thy-GFPm mice (The Jackson Laboratory) were bred onsite, and male and female C57BL/6 mice were birthed onsite from pregnant females obtained from The Jackson Laboratory. All animals were weaned at postnatal day 22 (P22) to P23 and housed in groups of four, separated by sex. All animals were handled $3 \mathrm{~d}$ priors to the start of training on P28. To minimize stress, handling consisted of scooping all animals in a single cage into the experimenter's hands at once for $1 \mathrm{~min}$, followed by each mouse being scooped and handled individually for $1 \mathrm{~min}$. Additionally, each animal was scruffed during the second handling session to habituate them to the handling associated with injections.

Drug

For actin-stabilizing imaging experiments, $200 \mathrm{~nm}$ jasplakinolide (Jasp; Tocris Bioscience) in $0.02 \%$ DMSO was bath applied to slices. During training, mice received $1 \mathrm{mg} / \mathrm{kg}$ doses of methamphetamine hydrochloride (Sigma-Aldrich). For systemic Blebb infusions mice received a 10 $\mathrm{mg} / \mathrm{kg}$, i.p., dose of racemic blebbistatin (TSRI) diluted to $1 \mathrm{mg} / \mathrm{kg}$ in a vehicle (Veh) of $0.9 \%$ DMSO/25\% hydropropyl $\beta$-cyclodextrin. Vehicle animals received the vehicle without Blebb. For intrahippocampal CA1 infusions, the active $(-)$ or inactive $(+)$ Blebb enantiomer (R\&D Systems) was infused at a concentration of $90 \mathrm{ng} / \mu \mathrm{l}$ in $10 \%$ DMSO and $0.9 \%$ saline. The delivery rate was $0.1 \mu \mathrm{l} / \mathrm{min}$ over $2 \mathrm{~min}$. Injectors were left in place for $1 \mathrm{~min}$ after completion of the infusion to allow for sufficient diffusion of drug away from the needle tips.

\section{Surgery}

Mice were anesthetized using isoflurane gas and implanted bilaterally with 22 guide cannulae (Plastics One) $1 \mathrm{~mm}$ above CA1 (anteroposterior, $-2 \mathrm{~mm}$ relative to bregma; $\mathrm{ML}, \pm 1.5 \mathrm{~mm}$ relative to bregma; $\mathrm{DV},-0.9$ $\mathrm{mm}$ from the skull). Metacam ( $1.5 \mathrm{mg} / \mathrm{ml}, 1-2$ drops orally) was administered immediately after surgery.

\section{Behavior}

Adolescent mice were trained as previously described (Young et al., 2017). Conditioned place preference (CPP) consisted of two phases, pretesting and training, followed by imaging $1-3 \mathrm{~d}$ later. Pretesting was conducted over 2 consecutive days. Animals received an intraperitoneal injection of saline before freely exploring all three CPP chambers for 30 min. Either the white or black chamber was assigned as the METH-paired chamber [conditioned stimulus (CS+)] for each animal based on their least preferred chamber during the final $15 \mathrm{~min}$ of the second pretest session. There were no initial differences between groups for the amount of time spent in either the white or black chamber. Over the next 4 consecutive days, animals were trained twice daily in 30 min training sessions. Animals either received saline in the CS chamber in the mornings and METH in the CS+ in the afternoons or were assigned the opposite training schedule. One to three days following the final day of training, animals were killed for acute tissue collection. After testing, the brains were postfixed overnight using $4 \%$ paraformaldehyde so that cannula tract location could be verified. Correct injector placement in the CA1 of the hippocampus was confirmed for all animals in the study.

\section{Spine motility}

Acute slice preparation. Acute coronal brain slices (350 $\mu \mathrm{m}$ thick) were extracted and sliced in cold cutting solution composed of the following (mм): 119 choline $\mathrm{Cl}, 22$ D-glucose, $4.3 \mathrm{MgSO}_{4} 2.5 \mathrm{KCl}, 1 \mathrm{NaH}_{2} \mathrm{PO}_{4}, 1$ $\mathrm{CaCl} 2$, and 26.2 NaHCO3. Adolescent animals (P28-P36) were gravity perfused with cold cutting solution for $40 \mathrm{~s}$ before brain extraction and slicing. Following slicing, brain slices were continuously bubbled with $95 \% \mathrm{O}_{2} / 5 \% \mathrm{CO}_{2}$ and incubated at $34^{\circ} \mathrm{C}$ for $30 \mathrm{~min}$ then room temperature for $30 \mathrm{~min}$ in artificial CSF ( $\mathrm{aCSF}$ ) composed of the following (mM): $119 \mathrm{NaCl}, 2.5 \mathrm{KCl}, 1.3 \mathrm{MgSO}_{4} 2.5 \mathrm{CaCl} 2,1 \mathrm{NaH}_{2} \mathrm{PO}_{4}, 0.2$ Trolox, 11 glucose, and 26.2 NaHCO3. For imaging, slices were continuously perfused with oxygenated aCSF at $1.5-2 \mathrm{ml} / \mathrm{min}$.

Two-photon imaging. Two-photon imaging of the basolateral amygdala complex (BLC) and CA1 was conducted with a multiphoton laserscanning microscopy (FV1000MPE-TWIN, Olympus), equipped with a water-immersion objective lens (ULTRA $25 \times$, numerical aperture 1.05, Olympus) and Fluoview software (Olympus). To excite eGFP, we used a Ti:sapphire laser and collected emitted photons that passed through a $500-550 \mathrm{~nm}$ bandpass filter with an external nondescanned detector. To measure spine dynamics, we imaged slices in XYZT dimension (45-60 $\mu \mathrm{m} z$-stack; 60 min time series; 5 min between stacks; Clement et al., 2012). The imaging parameters used were as follows: excitation $=930$ $\mathrm{nm}$; power at sample $=\sim 15 \mathrm{~mW}$; pixel dwell time $=2 \mu \mathrm{s} ; x-y$ scaling $=$ $0.201 \mu \mathrm{m} /$ pixel; and $z$-scale $=1 \mu \mathrm{m}$; no averaging. For the effects of actin stabilization, vehicle (aCSF with $0.02 \%$ DMSO) or aCSF + Jasp was bath applied to slices. Slices were allowed to stabilize in the imaging chamber and incubate for $30 \mathrm{~min}$ before imaging. Slices were then imaged in the XYZT dimension over $1 \mathrm{~h}$ with $5 \mathrm{~min}$ in between each $45-60 \mu \mathrm{m} z$-stack. Spine movement analysis. Spine movements were assessed in both the CA1 and BLC following standard procedure (Majewska and Sur, 2003; Clement et al., 2012). ImageJ software (NIH) was used to process and align images. After $z$-stack compression of the 12 images ( 5 min between 
Table 1. Comparison of initial dendritic spine length by age and region

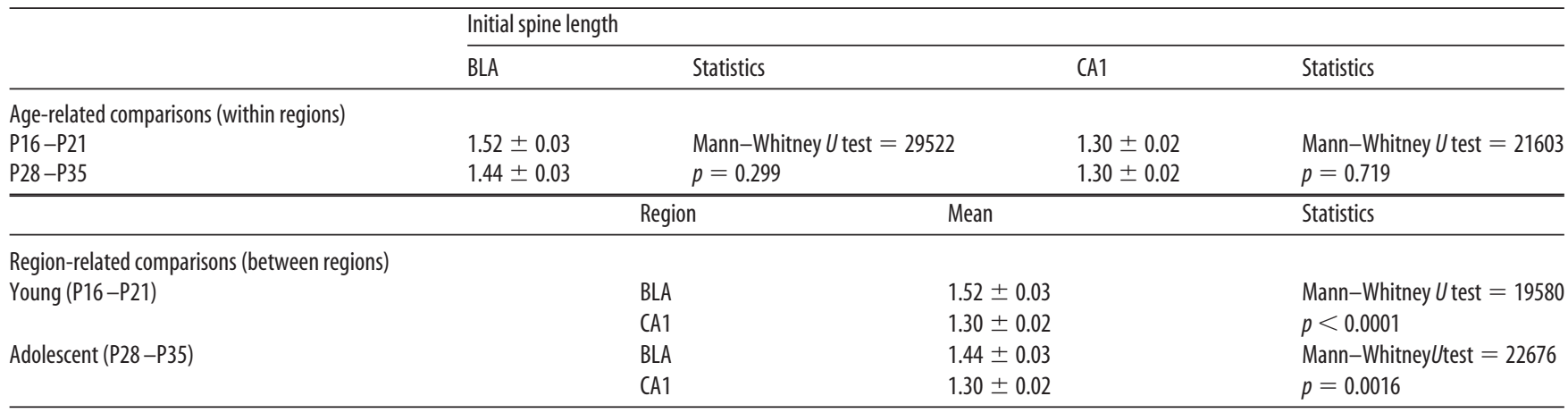

frames), the resulting two-dimensional projections were aligned to compensate for inherit $x-y$ drifting during time series imaging using the ImageJ plugin StackReg. Spines used for analysis were selected in an unbiased way with the researcher blinded to the group. Tertiary dendritic segments that were relatively clear of random axonic and dendritic processes were selected for analysis. Along these segments, the movement of the first 10 unobstructed dendritic spines was analyzed for a total of 30 spines per slice. The spine length was measured from the base to the tip of the protrusion in each of the 12 images. Spine movement was expressed as the average change in spine length per minute ( $\mu \mathrm{m} / \mathrm{min}$; Lendvai et al., 2000; Majewska and Sur, 2003; Clement et al., 2012).

\section{Statistical analysis}

For all studies, the experimenters were blind to the treatment group. The averages of spine movement per slice were found to be normally distributed but all spines per group was not. Therefore, comparisons of groups using slice averages was conducted using $t$ tests or oneway ANOVAs, while population comparisons were performed with Kolmogorov-Smirnov (KS) tests. For post hoc analysis of ANOVAs, Bonferroni's multiple-comparison test was used. To determine the presence of subpopulations within the data, for each study BLC and CA1 spine movements across all groups were combined and examined using cluster analysis. It was found that the data clustered unbiasedly into either two or three clusters. Dictating two or three clusters for the cluster analysis of each study had equal levels of good cluster quality. Visual examination of the data when grouped according to either the two or three cluster outputs revealed that three clusters modeled the data better than two clusters. Therefore, all data were analyzed using cluster analysis for three clusters. Cluster comparison within brain region was performed using $\chi^{2}$ analysis. A Mann-Whitney $U$ test or Kruskal-Wallis test was used to examine initial spine lengths. To examine the influence of highly motile spines on group differences, the receiver operating characteristic (ROC) was used to determine a cutoff of highly motile METH spines, which were then removed. Pretest training data were analyzed using Wilcoxon signed-rank tests.
A CA1 P16-21
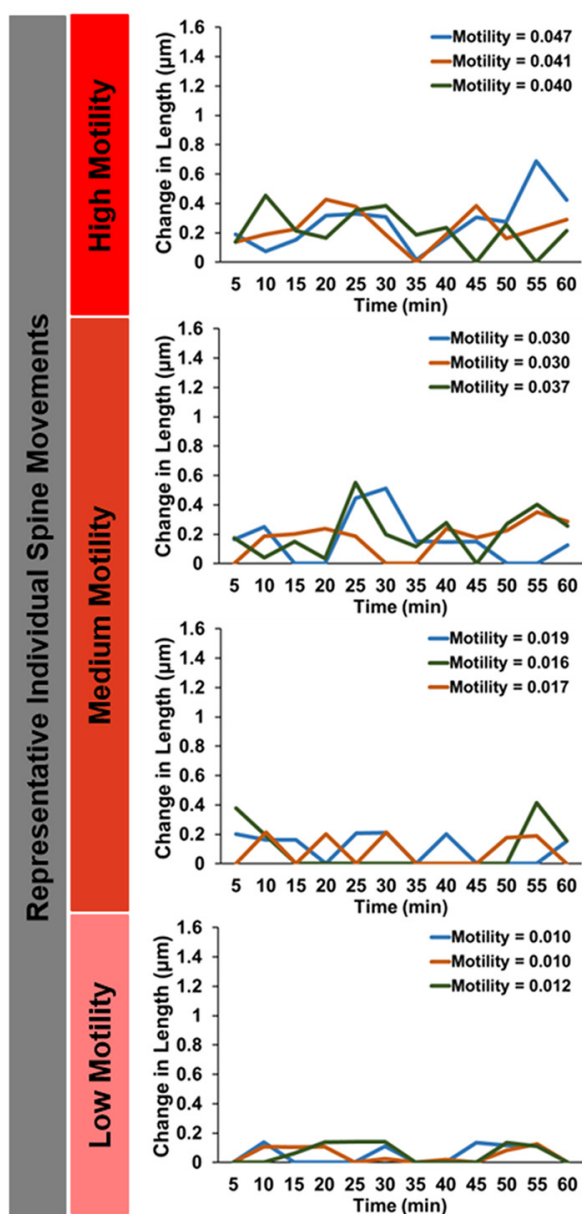

$5 \quad 1015202530354045505560$ B

BLA P16-21

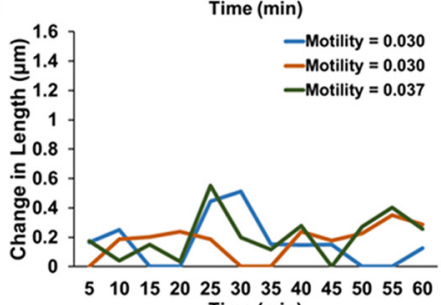

510152025303540
Time (min)

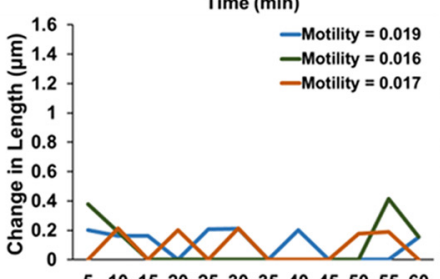

$5 \quad 1015202530 \quad 354045505560$ Time (min)
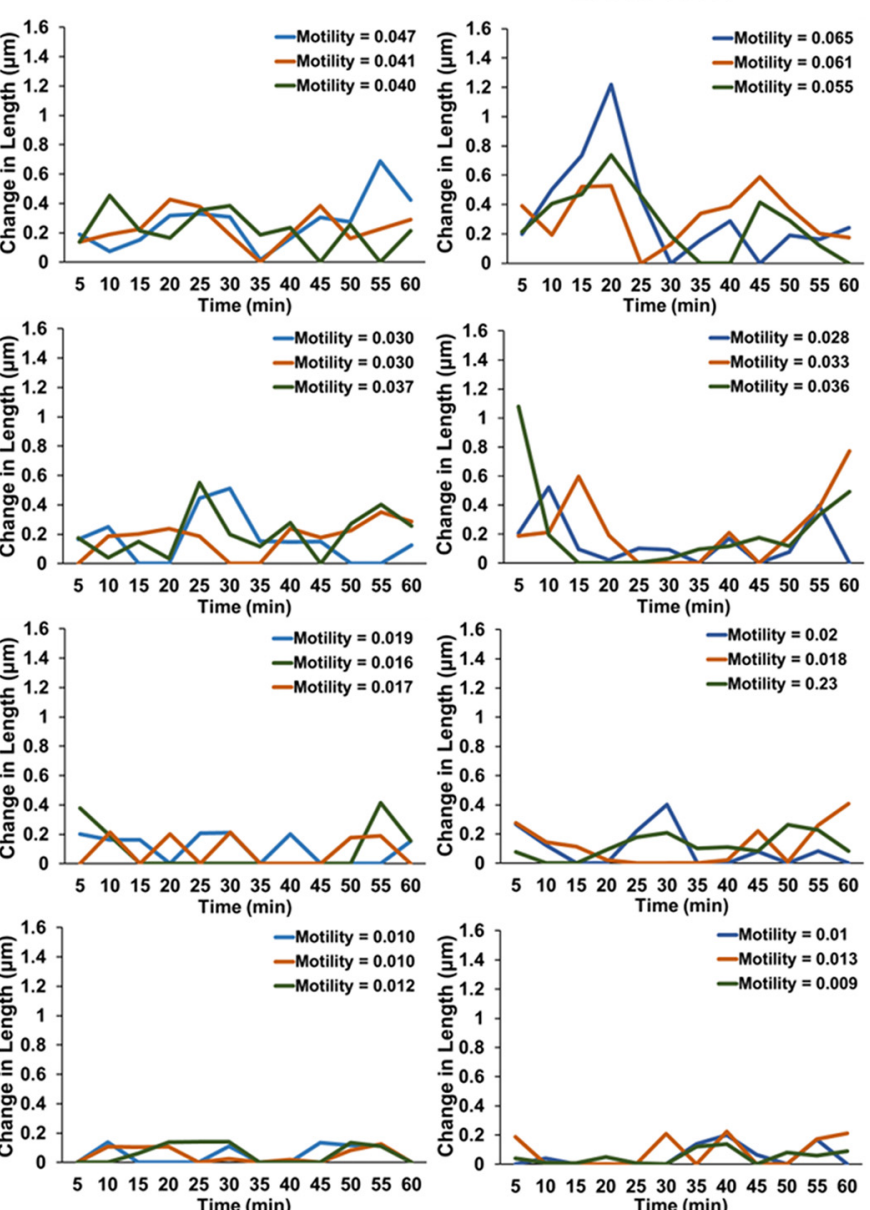

Time $(\mathrm{min})$

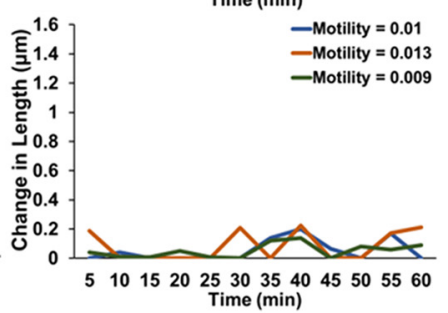

C Appearances and Disappearance of Spines

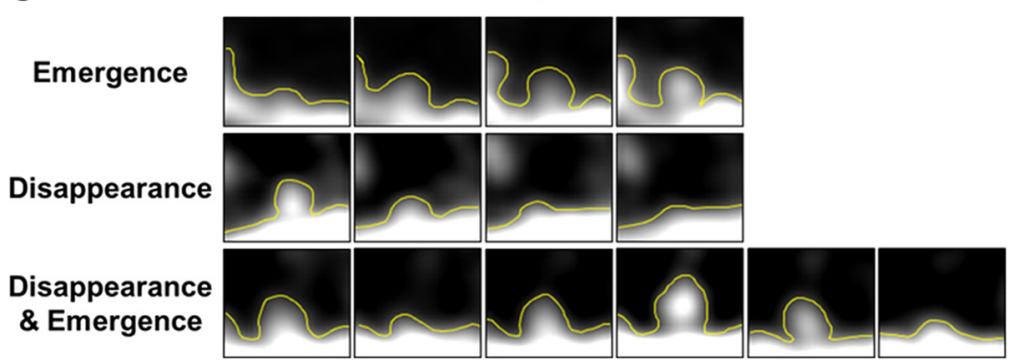

Figure 3. Age-related CA1 and BLA spine movements. $A, B$, Representative movements of individual CA1 ( $A$ ) and BLA (B) spines with different levels of motility over $1 \mathrm{~h}$. C, Examples of spines emerging and disappearing during the imaging period. 


\section{Results}

BLA and CA1 spine motility is actin dependent and decreases with development

Spine motility has not yet been assessed in the BLA. Therefore, we first examined age-dependent spine motility, as it has been reported to decrease with development elsewhere in the brain and likely acts as an important component of use-dependent synaptic wiring during development (Bonhoeffer and Yuste, 2002). The exact age at which spine motility decreases varies by brain region and preparation (Dunaevsky et al., 1999; Lendvai et al., 2000; Majewska and Sur, 2003; Clement et al., 2012). Therefore, we examined spine movements across two postnatal day ranges, $\mathrm{P} 16-\mathrm{P} 21$ and P28-P35. Acute slices from naive Thy1-GFP $(\mathrm{m})$ mice, containing both CA1 and BLA, were imaged every 5 min for $1 \mathrm{~h}$. The motility of 30 spines per slice was then quantified. Consistent with prior reports on spine motility in the hippocampus (Dunaevsky et al., 1999; Lordkipanidze et al., 2013), CA1 spines displayed more motility at P16-P21, compared with P28-P35 (Fig. $1 A ; t_{(12)}=3.77, p<0.01$; power $=0.93$ ). Before performing any additional analyses, we confirmed that the range of spine motilities observed as a group was represented across spines from all slices analyzed and was, therefore, not biased by a slice with particularly high or low overall motility (Fig. 1B). This was performed for all experiments in the study. To determine whether the shift in spine movements was driven by decreased motility across the whole population of spines or by loss of a subgroup of spines only present in P16-P21 CA1, we examined the cumulative distribution of spine movement. This revealed a shift in the whole population of spines toward decreased movement at P28$\mathrm{P} 35$, as well as the loss of highly motile spines (movement, $\geq 0.030 \mu \mathrm{m} / \mathrm{min}$; Fig. $1 C$; KS test, $p<0.0001$ ). BLA spine movements followed a similar developmental pattern (Fig. $1 D ; t_{(14)}=$ $5.01, p<0.001$, power $=0.99$ ), with an even greater shift in the population of spines toward decreased motility at P28-P35 (Fig. $1 F$; KS test, $p<0.0001)$.

We next performed unbiased cluster analysis, identifying three clusters. This enabled comparison of the relative representation of spines in low-, mid-, and high-range motility within CA1 and BLA in early life (P16-P21) versus early adolescence (P28-P35; combined overall: $\chi_{(6)}^{2}=95.93, p<0.0001$; CA1 overall: $\chi_{(2)}^{2}=20.30, p<0.0001$; BLA overall: $\chi_{(2)}^{2}=65.82, p<$ 0.0001 ; Fig. $2 A) \cdot \chi^{2}$ analysis established age-dependent differences in CA1 for low (cluster 1) and mid-range (cluster 2) motility (cluster 1: $\chi_{(1)}^{2}=20.15, p<0.0001$; cluster $2: \chi_{(1)}^{2}=19.14, p<$ 0.0001 ; cluster $3: \chi_{(1)}^{2}=1.00, p>0.05$; Fig. $\left.2 B\right)$, while motility decreased in all three clusters (including high motility) with age for BLA spines (cluster 1: $\chi_{(1)}^{2}=65.64, p<0.0001$; cluster $2: \chi_{(1)}^{2}$ $=57.43, p<0.0001$; cluster $3: \chi_{(1)}^{2}=6.58, p<0.01$; Fig. $\left.2 C\right)$. When comparing regions, BLA spines were more motile than CA1 spines during early life (P16-P21; KS test, $p<0.05)$, while the opposite was true in early adolescence (P28-P35; KS test, $p<$ 0.0001; Fig. $1 G, H)$. Additional similarities and differences were observed between CA1 and BLA. The most pronounced difference was in initial spine lengths. BLA spines were longer than those in CA1, and this was not age dependent (Table 1). This is consistent with a prior report using Golgi staining, though a direct comparison was not made in that study (Luczynski et al., 2016). Examining individual spines in early development (P16P21), a time point when motility was sufficiently elevated to reveal variability in movements between spines over the course of an hour, we found CA1 and BLA spines with low to medium overall motility displayed a remarkably similar range of move-
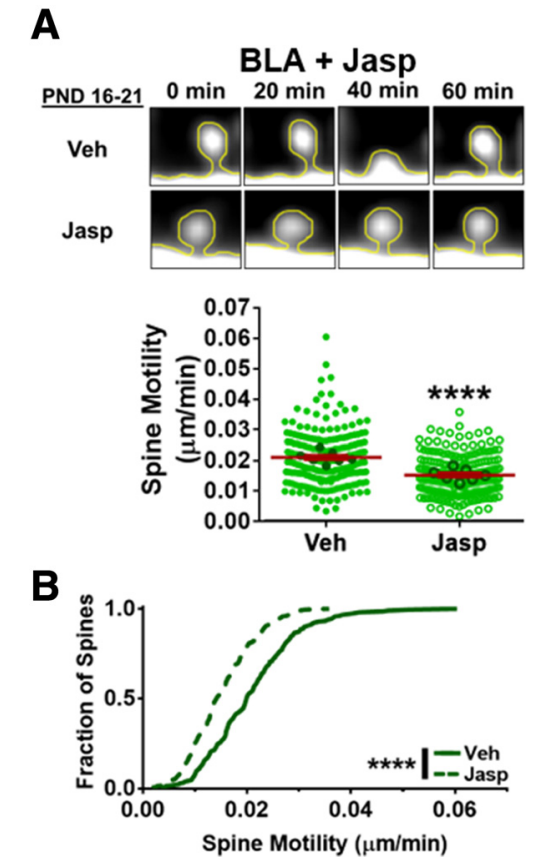

Figure 4. Actin stabilization of young BLA spines. A, Young (P16-P21) BLA slices were incubated in bath-applied Veh or Jasp for 30 min before imaging. $\boldsymbol{B}$, Spine movements and cumulative distributions were measured in the presence of Jasp or Veh (Veh: $n=210$ spines, 7 slices, 5 animals; Jasp: $n=210$ spines, 7 slices, 6 animals). Error bars represent SEM. ${ }^{* * *} p<$ 0.0001 .

ment events (Fig. $3 A, B$ ). These movements were relatively infrequent and represented small to medium changes in length, rather than, for example, only one to two large movements that averaged to moderate motility over the course of an hour. Differences emerged in spines with overall high motility rates; BLA spines made larger, more frequent movements when compared with CA1 spines (Fig. $3 A, B$, top). In addition, the appearance and disappearance of spines over the course of imaging, while rare, was observed in both regions (Fig. 3C). It was more prominent in young BLA slices and CA1 slices from both age ranges, occurring in $3 \%$ of spines.

Spine motility is actin dependent in the hippocampus and cortex (Fischer et al., 1998, 2000). To confirm that our measurements were a readout of actin-dependent processes within dendritic spines, we measured the effect of the actin stabilizer Jasp on BLA spine dynamics in slices from P16-P21 animals. Indeed, Jasp decreased BLA spine movement (Fig. $4 A, B ; t_{(12)}=5.48, p=$ 0.0001 ; Power $=0.99$; KS test, $p<0.0001)$. This included an absence of highly motile spines, making it more akin to P28-P35 spine motility distribution (Fig. $1 F$ ). We also confirmed there were no group differences in initial spine length for this and all subsequent experiments (Tables 1,2). Together, these data establish that BLA spine motility is actin dependent and decreases markedly by P28-P35, with a significant loss of highly motile spines $(\geq 0.03 \mu \mathrm{m} / \mathrm{min})$.

\section{METH conditioning increases basal spine motility in BLA, but not CA1}

We next assessed BLA spine motility following METH-associated learning as a measure of spine actin dynamics in male and female Thy1-GFP(m) mice. The number of neurons expressing fluorescence increases dramatically with development and, accordingly, we found that P28-P35 is the oldest age at which it is possible to confidently resolve individual dendritic segments and associated 


\begin{tabular}{|c|c|c|c|c|c|}
\hline & & \multicolumn{4}{|c|}{ Initial spine length } \\
\hline & & BLA & $\begin{array}{l}\text { Statistics (Mann-Whitney } \\
\text { or Kruskal-Wallis test) }\end{array}$ & CA1 & $\begin{array}{l}\text { Statistics (Mann-Whitney } \\
\text { or Kruskal-Wallis test) }\end{array}$ \\
\hline Jasp slice treatment & Veh & $1.56 \pm 0.03$ & $U=20724$ & & \\
\hline \multirow[t]{2}{*}{ METH CPP } & Saline & $1.48 \pm 0.03$ & $U=28698$ & $1.21 \pm 0.02$ & $U=29214$ \\
\hline & METH & $1.48 \pm 0.03$ & $p=0.947$ & $1.26 \pm 0.02$ & $p=0.055$ \\
\hline \multirow[t]{2}{*}{ METH CPP + Blebb treatment } & Saline/Veh & $1.66 \pm 0.03$ & $H(3)=7.38$ & $1.22 \pm 0.02$ & $H(3)=5.89$ \\
\hline & METH/Blebb & $1.59 \pm 0.03$ & & $1.30 \pm 0.02$ & \\
\hline
\end{tabular}

spines in the BLA. Importantly, we have previously demonstrated that METH-associated memory is vulnerable to disruption by Blebb days after training in both adult and adolescent (P28-P35) male and female mice (Young et al., 2017).

Mice underwent CPP training with either saline or METH, followed by imaging 1-3 d later (Fig. 5A). Behavioral testing was not performed before imaging to avoid retrieval and/or extinction-induced effects on spine motility. However, our CPP training protocol consistently produces a conditioned place preference for METH in adolescents (Young et al., 2017; see Fig. 12). Similar to P16-P21 motility in naive slices (Fig. 3), differences in individual spine movements were the most pronounced as motility increased (Fig. 6A,B). Additionally, as in naive slices, the appearance and disappearance of spines over the course of imaging was rare following CPP training (1.7-2.9\%) and was not influenced by METH exposure.

Spine motility was elevated in the BLA, but not in CA1, 1-3 d after METH training relative to saline controls (Fig. $5 B, C$; BLA: $t_{(15)}=2.38, p<0.05$, power $=0.60$; CA1: $t_{(16)}=1.27, p>0.05$, power $=0.22$ ), and motility was equally represented across slices analyzed (Fig. 6C,D). Moreover, every slice imaged from METHtrained animals had spines in the BLA that reached the "highmotility" threshold of $\geq 0.03 \mu \mathrm{m} / \mathrm{min}$ (Fig. $6 C$ ), identified in the age-dependent motility experiment in Figure 1. As a reminder, the BLA and CA1 of young (P16-P21), but not adolescent (P28P35) animals displayed spine motility rates $>0.03 \mu \mathrm{m} / \mathrm{min}$ (Fig. $1 D-F)$. High-motility spines were not observed in the BLA from the saline condition (Fig. $6 C$ ) or in CA1 following saline or METH training (Fig. 6D). Further analysis of spine motility with a KS test of the cumulative distribution of spines revealed that METH training shifted all spine movements in the BLA $(p<$ $0.0001)$, but not in CA1 $(p=0.06)$, toward higher motility and precipitated the appearance of highly motile spines (Fig. $5 D, E$ ). Even with highly motile spines identified and removed using ROC to establish a cutoff criteria, METH training still significantly increased BLA spine movements (BLA: $p<0.0001$, power $=1.0$; CA1: $p>0.05$, power $=0.82$; Fig. $5 F, G)$. Thus, the effect of METH on BLA spine dynamics cannot be attributed solely to a subset of highly dynamic spines. However, given the sparse distribution of memories, it is plausible that METHassociated memory is preferentially targeted to highly motile spines.

To examine motility in greater depth, cluster analyses were performed (combined overall: $\chi_{(6)}^{2}=33.29, p<0.0001$; BLA overall: $\chi_{(2)}^{2}=15.07, p<0.001$; CA1 overall: $\chi_{(2)}^{2}=6.58, p<$ 0.05 ; Fig. $7 A$ ), as in Figure 2. $\chi^{2}$ analysis showed that METH decreased the number of spines in the low-motility group (cluster 1: $\left.\chi_{(1)}^{2}=9.25, p<0.01\right)$ and increased the number of spines in the high-motility group (cluster $3: \chi_{(1)}^{2}=10.59, p<0.001$ ), relative to spines from saline-treated mice (cluster $2: \chi_{(1)}^{2}=3.33$, $p>0.05$; Fig. $7 B$ ). In contrast, the high-motility cluster 3 I CA1 was unchanged by METH training (cluster 1: $\chi_{(1)}^{2}=5.59, p<$ 0.05 ; cluster $2: \chi_{(1)}^{2}=4.11, p<0.05$; cluster $3: \chi_{(1)}^{2}=2.20, p>$ 0.05 ; Fig. $7 C$ ). Interestingly, BLA spine movement appeared to be restricted to the spine head, as very little neck motility was observed in spines, regardless of treatment during training, and it did not correlate with the overall motility rate of the spine $\left(F_{(3,116)}\right.$ $=20.61, p<0.0001$; neck saline vs METH, $p>0.05$; overall length saline vs METH, $p<0.01$; Fig. 8). Collectively, these data indicate that $\mathrm{METH}$-associated learning produces a selective and lasting increase in basal spine actin dynamics (i.e., no acute stimulation at the time of imaging) of P28-P35 BLA spine heads that are normally quite stable.

\section{Persistent METH-induced BLA spine motility is NMII dependent}

To directly test the hypothesis that METH-induced BLA spine dynamics are sustained by NMII activity, we next measured the effect of Blebb on BLA and CA1 spine motility. Animals underwent saline or METH-associated CPP training, as in Figure 4. One to three days later, and $24 \mathrm{~h}$ before imaging, mice were treated with either Blebb or vehicle intraperitoneally, resulting in four groups (Saline/Veh, Saline/Blebb, METH/Veh, METH/ Blebb; Fig. 9A). Comparing the spine motility of saline and METH-trained animals given vehicle $1-3$ d post-training replicated the results reported in Figure 4. Specifically, METH conditioning increased the overall motility of spines in the BLA, but not in CA1 (BLA: $F_{(3,25)}=6.69, p<0.01 ; \mathrm{METH} /$ Veh vs Saline/Veh, Saline/Blebb, METH/Blebb: $p<0.001$, power $=0.95$; CA1: $F_{(3,24)}$ $=0.95, p>0.05$, power $=0.23$; Fig. $9 B, C)$. The cumulative distribution of spines followed a similar pattern, with a large shift in BLA motility and increased representation of highly motile spines (Fig. 9D, top right; Saline/Veh vs METH/Veh, $p<0.0001$ ). CA1 spines showed a small, but significant, shift in the distribution of motility, but no appearance of highly motile spines (Fig. $9 E$, top right; Saline/Veh vs METH/Veh, $p<0.05)$. Again, all effects were broadly consistent across slices (Fig. 10). Using a protocol that disrupts METH-associated memory (intraperitoneal Blebb 1-3 d post-training; Young et al., 2017), we observed a reversal of METH-associated increases in BLA spine movements (Figs. 9B,D, bottom left, 10; METH/Veh vs METH/Blebb, $p<$ $0.0001)$. As a result, the distribution of spine motility for the METH/Blebb group was identical to BLA spines from the Saline/ Blebb group (Fig. 9D, bottom right; Saline/Blebb vs METH/ Blebb, $p>0.05)$. Blebb had no effect on the motility of BLA or CA1 spines from saline-trained animals (Fig. $9 B, C$, and $D, E$, top left; BLA: Saline/Veh vs Saline/Blebb, $p>0.05$; CA1: Saline/Veh vs Saline/Blebb, $p>0.05)$. While Blebb had no effect on overall spine motility in CA1 (Fig. 9C), it did reverse the small rightward shift present in METH-trained, vehicle-treated mice (Fig. 9E, 
A

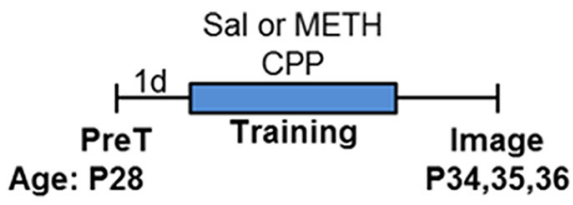

B

BLA

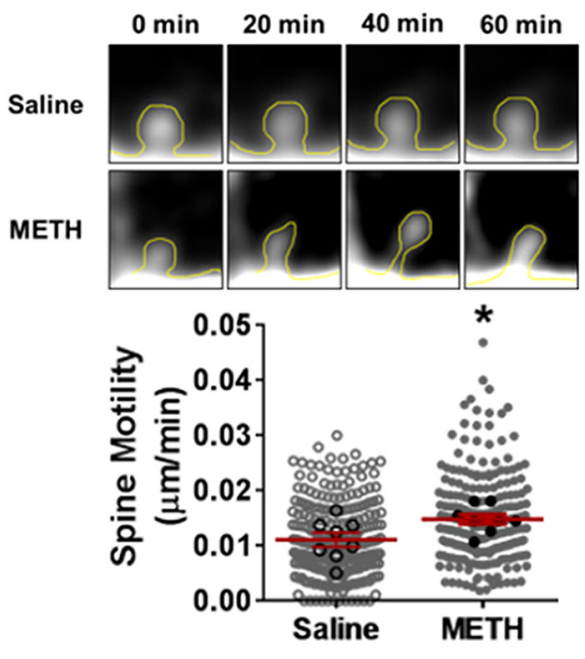

D

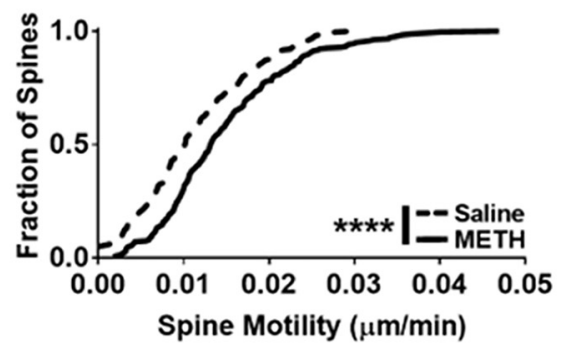

F

G
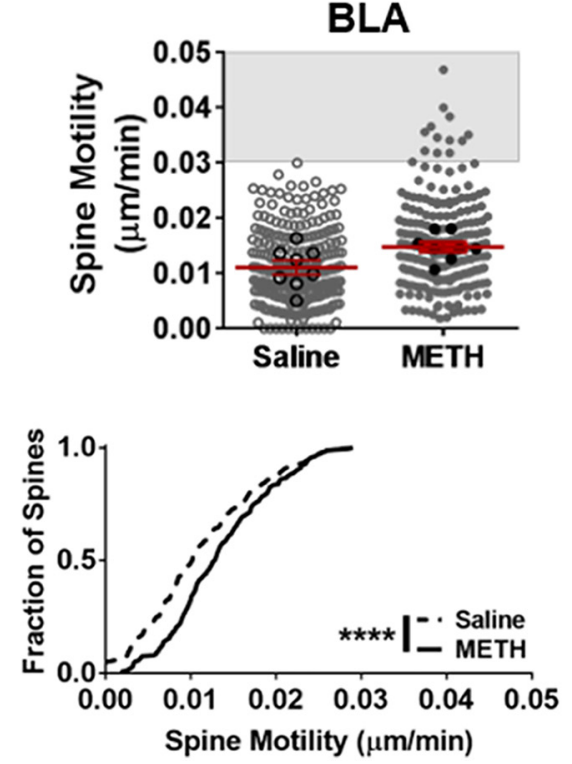

C
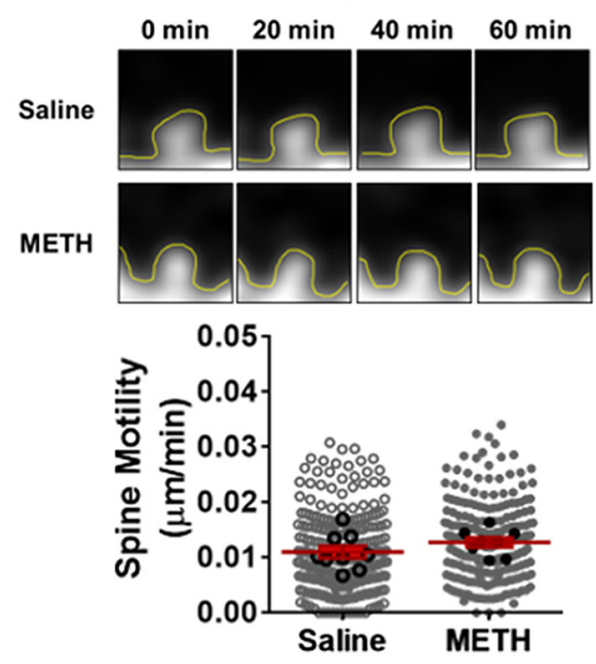

E

CA1
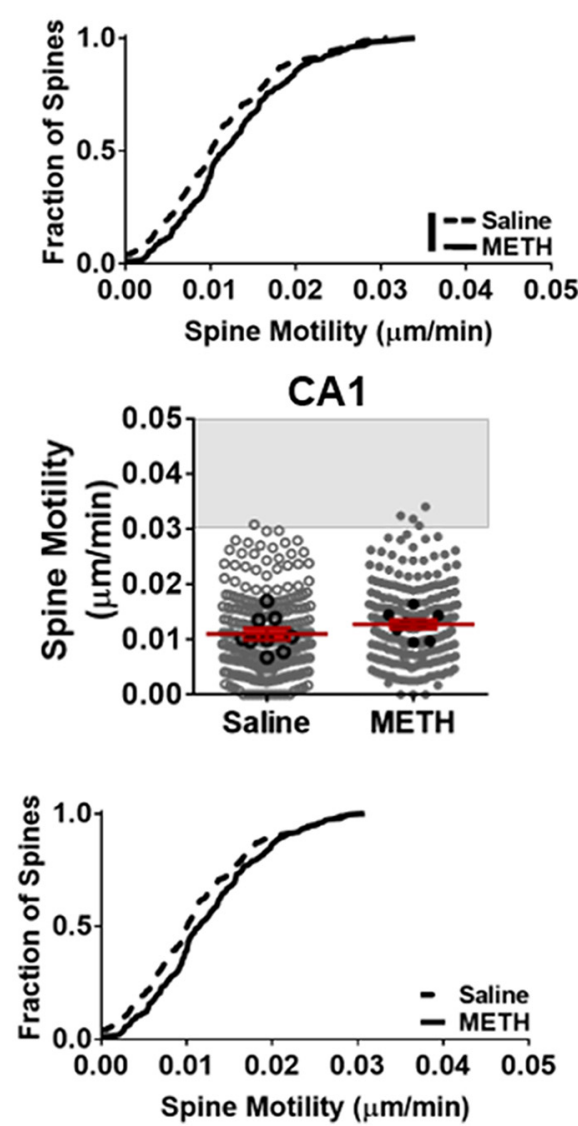

Figure 5. METH conditioning produces a persistent increase in spine motility in BLA, but not in CA1. $A$, Schematic of the experimental design. $B, C$, Spine movements in the BLA (B) and CA1 (C) were measured over $1 \mathrm{~h}, 1-3 \mathrm{~d}$ after saline or METH training. Small circles represent the movement of each spine, while the larger circles with heavier borders are the average of 30 spines per slice (BLA: saline, $n=240$ spines, 8 slices, 8 animals; METH, $n=240$ spines, 8 slices, 7 animals; CA1: saline, $n=270$ spines, 9 slices, 7 animals; METH, $n=240$ spines, 8 slices, 6 animals). D, E, Cumulative distributions of spine movements in the BLA (D) and CA1 (E) following training. $\boldsymbol{F}$, ROC curves were used to determine cutoff for highly motile spines (those in the gray boxes). The cutoff was set as the maximum motility displayed by any spine from the saline-treated condition $(0.030 \mu \mathrm{m} / \mathrm{min}) . \mathbf{G}$, Cumulative distributions of spine movements with highly motile spines removed. Error bars represent SEM. ${ }^{*} p<0.05,{ }^{* * * *} p<0.0001$. 
A

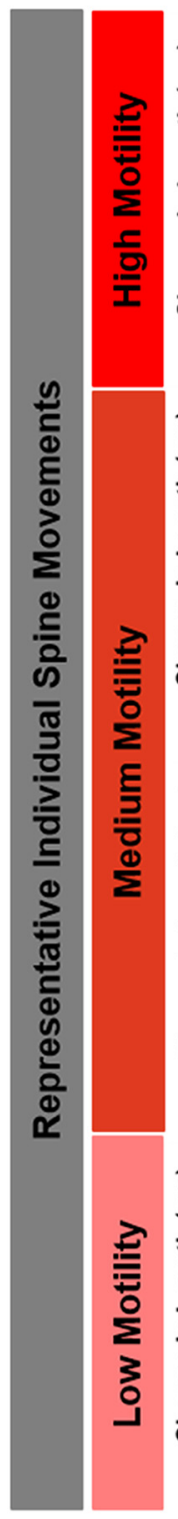

BLA

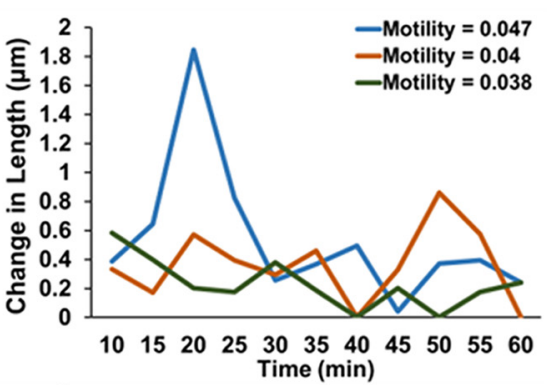

B
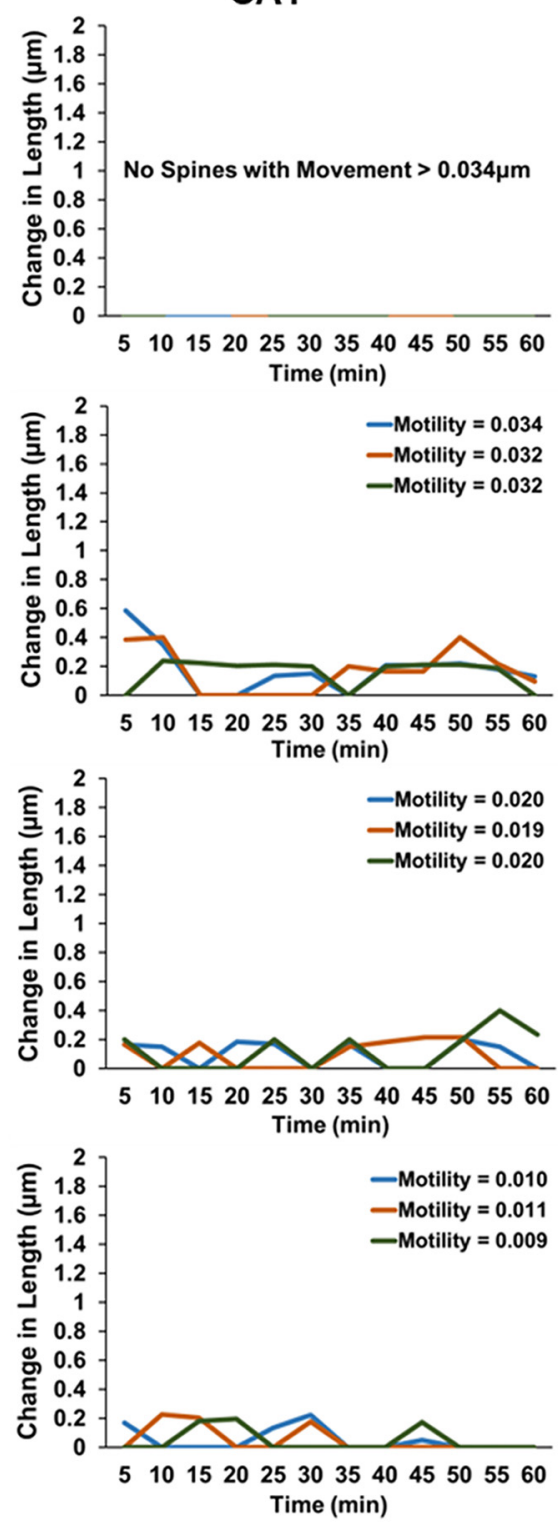

D
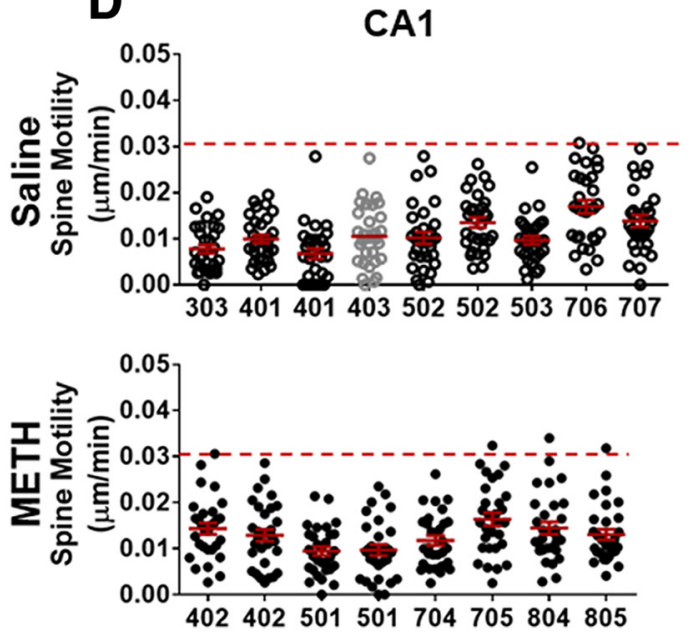

Figure 6. BLA and CA1 spine movements following CPP training. $A, B$, Representative movements of individual $(\boldsymbol{A})$ BLA and $(\boldsymbol{B})$ CA1 spines with different levels of motility over $1 \mathrm{~h}$. C, $\boldsymbol{D}, \mathrm{BLA}(\boldsymbol{C})$ and $C A 1(D)$ spine movements by slice and animal. Lighter colored circles denote females, and darker colored circles denote males. 
A

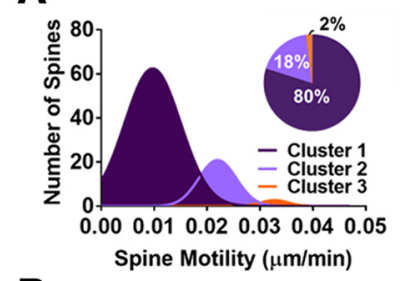

B

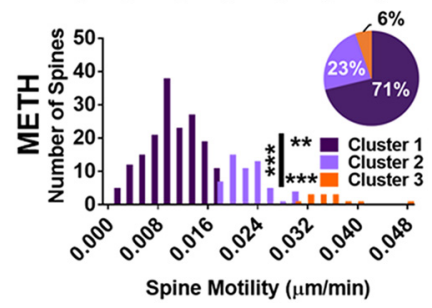

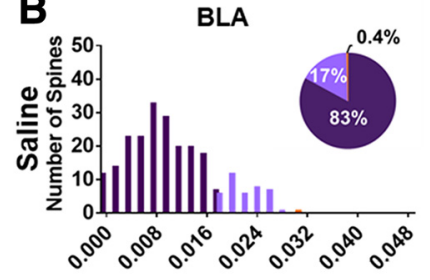

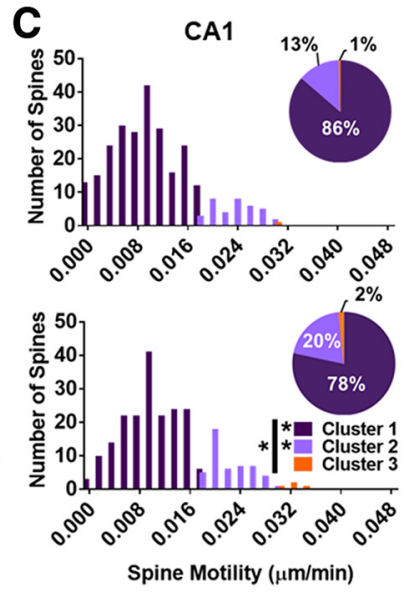

Figure 7. Cluster analysis of spine motility following CPP training. $A$, All BLA and CA1 spines underwent cluster analysis. $\boldsymbol{B}, \boldsymbol{C}$, Number of spines in each cluster for BLA ( $\boldsymbol{B})$ and CA1 ( $\boldsymbol{C}$ ) spines. Error bars represent SEM. ${ }^{*} p<0.05,{ }^{* *} p<0.01,{ }^{* * *} p<0.001, \chi^{2}$ analyses.

bottom left; METH/Veh vs METH/Blebb, $p>0.05)$. This is further supported by cluster analysis (Fig. 11), which revealed that METH training influenced all three clusters in the BLA, including the development of highly motile cluster 3 and that this population of spine motility was almost entirely lost with Blebb (Figs. 9F, 11; BLA overall: $\chi_{(6)}^{2}=63.68, p<0.0001$; Saline/Veh vs Saline/ Blebb overall: $\chi_{(2)}^{2}=0.01, p>0.05$; Saline/Veh vs METH/Veh overall: $\chi_{(2)}^{2}=34.63, p<0.0001$; cluster $1: \chi_{(1)}^{2}=26.60, p<$ 0.0001 ; cluster $2: \chi_{(1)}^{2}=13.17, p<0.001$; cluster $3 ; \chi_{(1)}^{2}=16.63$, $p<0.0001$; METH/Veh vs METH/Blebb overall: $\chi_{(2)}^{2}=23.25$, $p<0.0001$; cluster $1: \chi_{(1)}^{2}=17.89, p<0.0001$; cluster $2: \chi_{(1)}^{2}=$ 8.51, $p<0.01$; cluster 3: $\chi_{(1)}^{2}=11.23, p<0.001$; Saline/Blebb vs METH/Blebb: $\left.\chi_{(2)}^{2}=3.32, p>0.05\right)$. The most notable difference between the BLA and CA1 following METH training was the complete absence of highly motile spines in CA1 (Figs. $7 B, C$, $9 F, G$; CA1 overall: $\chi_{(6)}^{2}=7.24, p=0.06$; Saline/Veh vs Saline/ Blebb: $\chi_{(2)}^{2}=0.05, p>0.05$; Saline/Veh vs METH/Veh: $\chi_{(2)}^{2}=$ 5.34, $p<0.05$; METH/Veh vs METH/Blebb: $\chi_{(2)}^{2}=3.86, p<$ 0.05; Saline/Blebb vs METH/Blebb: $\left.\chi_{(2)}^{2}=0.01, p>0.05\right)$.

We previously shown that systemic intraperitoneal NMII inhibition disrupts METH-associated memory in adult animals (Young et al., 2014, 2016). This disruption is mediated through a selective effect within the BLA, as direct infusions into the BLA, but not CA1, the nucleus accumbens, or the orbital frontal cortex, recapitulate the ability of systemically administered Blebb to disrupt METH-associated memory in adult animals (Young et al., 2014, 2016; Briggs et al., 2017). Similar to adults, we have found that METH-associated memory in adolescent animals, which this study used for technical reasons, is similarly disrupted by systemic NMII inhibition (Young et al., 2017). The spineimaging data presented support the prediction that intra-CA1 Blebb infusions in adolescents should have no effect on the memory, similar to adults. Indeed, Blebb infused into CA1 of adolescent mice 30 min before testing (Fig. 12 A, B, Test 1) had no effect

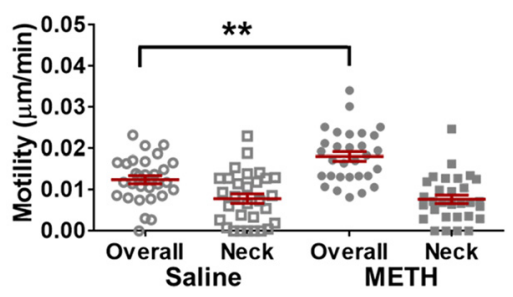

Figure 8. Comparison of neck motility following training. Neck motility was measured in a subset of high- and medium-motility spines from saline- and METH-treated animals. Error bars represent SEM. ${ }^{* *} p<0.01$.

on the METH-associated memory then or $24 \mathrm{~h}$ later (Fig. 12, Test 2; Veh overall: $F_{(2,27)}=17.18, p<0.0001$; PreTest vs test $1, p<$ 0.001; PreTest vs test 2, $p<0.0001$; Blebb overall: $F_{(2,24)}=4.86$, $p<0.05$; PreTest vs test $1, p<0.05$; PreTest vs test $2, p<0.05$ ). This provides indirect support for the particular importance of the highly motile spines, which resemble early postnatal spine movements and are present in the BLA but not CA1 after METH training, to the storage of $\mathrm{METH}$-associated memory and its unique vulnerability to NMII inhibition.

\section{Discussion}

We have previously reported that direct actin depolymerization or NMII inhibition within the BLA results in an immediate, longlasting and retrieval-independent disruption of METH-associated memory and reversal of METH-induced spine density increases to basal levels (Young et al., 2014, 2016). Interestingly, NMII inhibition within CA1 does not produce the same retrievalindependent disruption of METH-associated memory, nor does it alter METH-induced increases in spine density. Here, we used time-lapse two-photon imaging of spine motility to begin to address the hypothesis that the selective susceptibility of METHassociated memory and spines to NMII inhibition is due to NMII-dependent actin dynamics in BLA spines that uniquely persist well past the acute phase of METH-associated learning.

Because spine motility had not been assessed in the BLA before, we first confirmed that motility rates decrease with age, as has been reported in cultured hippocampal cells and slices, acute dentate gyrus and CA1 slices, and primary visual cortex in vivo (Fischer et al., 1998; Dunaevsky et al., 1999; Lendvai et al., 2000; Parnass et al., 2000; Portera-Cailliau et al., 2003; Clement et al., 2012; Lordkipanidze et al., 2013). This also allowed us to compare and deeply characterize spine motility in the BLA and CA1 in early postnatal life and later, in adolescence. BLA spines, which are longer than CA1 spines, were more motile than CA1 at P16$\mathrm{P} 21$. This is not overly surprising, as the amygdala undergoes intense innervation between P18 and P20 (Bouwmeester et al., 2002; Brummelte and Teuchert-Noodt, 2006), a process that occurs earlier in the hippocampus (P14-P16; Morris, 1985). Therefore, it is possible that more similar spine movements would be observed if P16-P21 BLA spines were compared with P14-P16 CA1 spines. At P28-P35, as developmental synaptic refinement is ending, spine motility dramatically decreased in both BLA and CA1. Interestingly, when directly comparing motility rates between the BLA and CA1 at P28-P35, BLA spines were more stable, making the subsequent impact of METH training on BLA motility even more striking. We also confirmed that BLA spine motility is actin dependent, as has been shown in cultured hippocampal cells and slices (Fischer et al., 1998; Dunaevsky et al., 1999; Parnass et al., 2000; Lordkipanidze et al., 2013). This established that spine motility is an appropriate measure to determine 
A

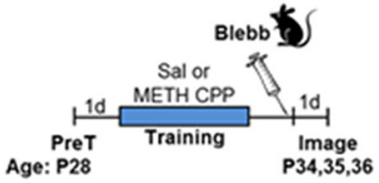

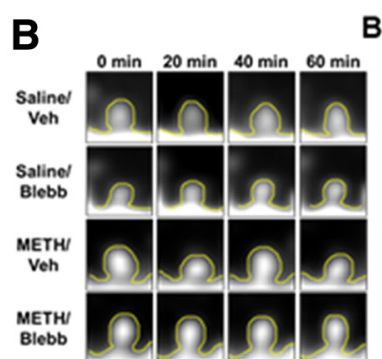

BLA

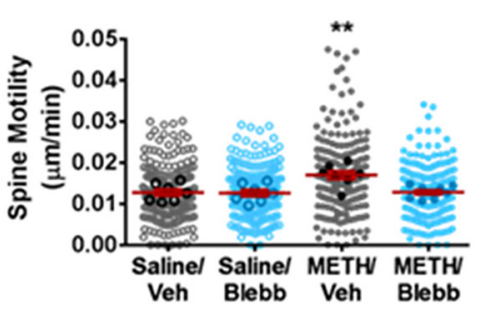

D
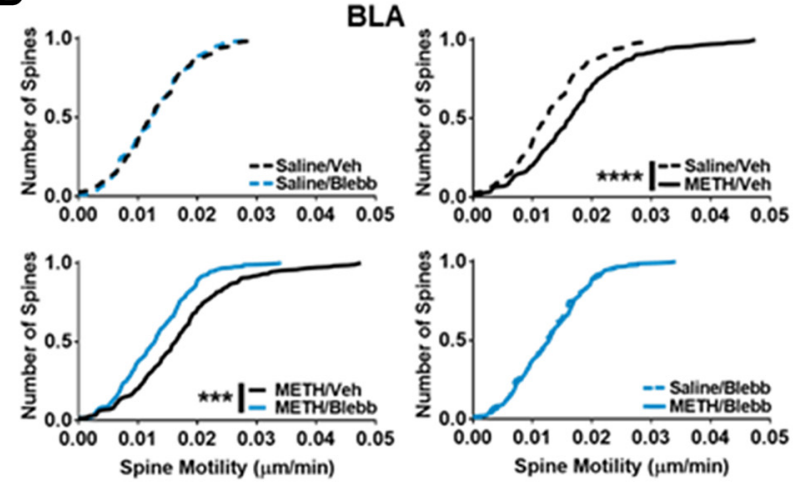

F

$\underset{\star \text { BLA }}{\star \star \star \star *}$

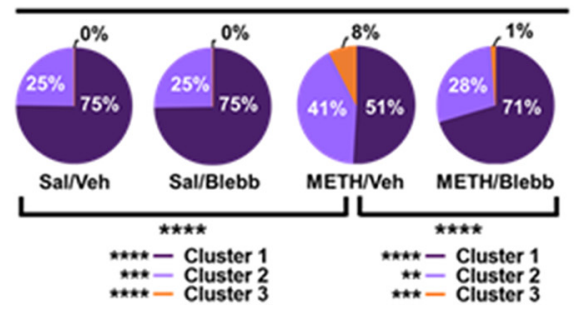

C

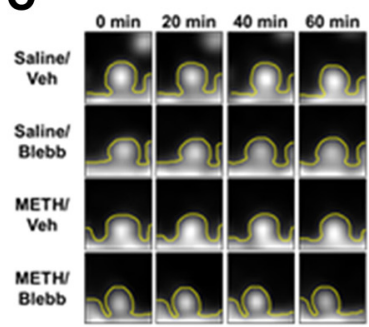

E
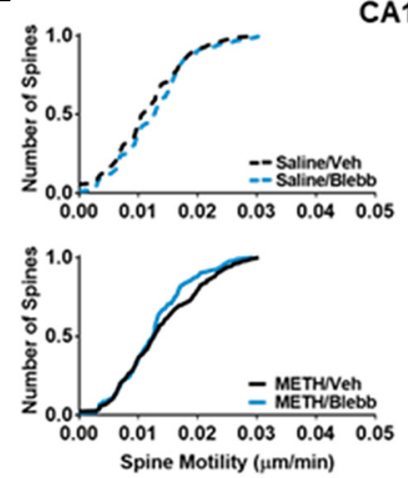

G

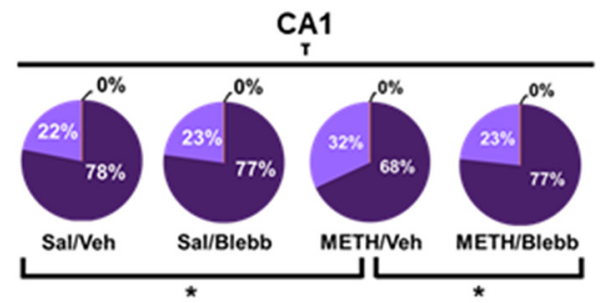

CA1

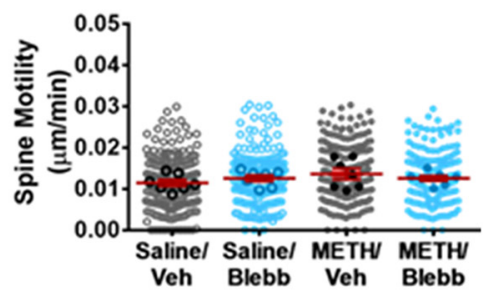

CA1
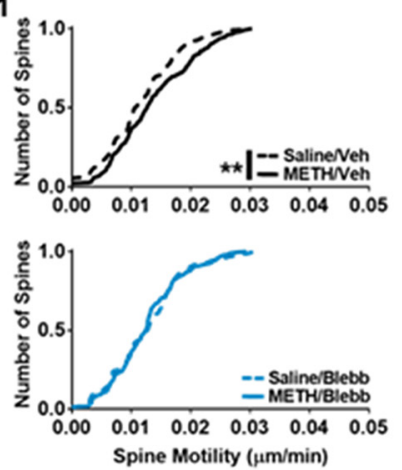

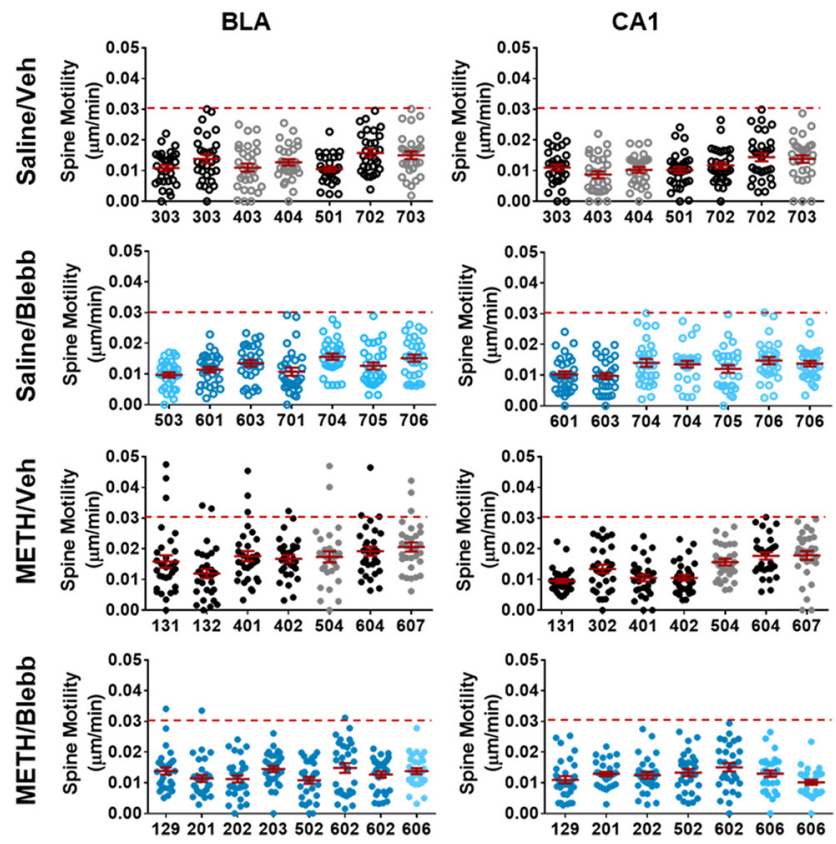

Figure 10. CA1 and BLA spine motility across different slices and animals. One to two slices were used per animal, and the movement of 30 spines per slice was analyzed.

memory stability. In addition, while it is not yet technically possible to do so, we are very interested in selectively ablating these highly motile spines to determine whether this is sufficient to disrupt METH-associated memory, which would be consistent with the sparsely distributed nature of memory.

Deeper analysis revealed that the spine movements were restricted to the head, as there was no change in spine neck motility. Spine neck motility has been connected to functional changes in spines through the regulation of calcium entry and exit (Araya et al., 2006b), suggesting that such dynamic changes are not occurring within BLA spines altered by METH training. This is consistent with the notion that the persistent motility in response to METH training does not reflect a process actively involved in METH-associated memory maintenance. Rather, we favor the interpretation that METH-associated memory persists despite this lack of normal actin-myosin stabilization and that this aberrant plasticity imparts a unique vulnerability of METHassociated memory to disruption long after learning. This is the foundation of our emerging therapeutic strategy to reduce relapse to METH seeking (Young et al., 2015, 2016).

NMII inhibition disrupts METH-associated memory and reverses BLA spine density with equal efficacy to direct actin depolymerization (Young et al., 2014, 2016, 2017; Briggs et al., 2017). If the persistent BLA spine motility changes observed here are connected to the memory disruption, we would predict the motility to be NMII dependent. And indeed, NMII inhibition reverted BLA spine motility to control levels, without influencing CA1 motility. In addition, we replicated the effects of METH training on BLA motility reported in Figure 4. It is important to note that Blebb had no effect on BLA spine motility under control (saline-treated) conditions. It is possible that this reflects a dosedependent effect; that is, a higher concentration of Blebb would disrupt motility. Higher doses of Blebb are not tolerated by animals, preventing us from directly testing this possibility. However, we are currently developing Blebb analogs with improved safety profiles that can be used to address this in the future. An alternative explanation to the possibility of a dose-dependent

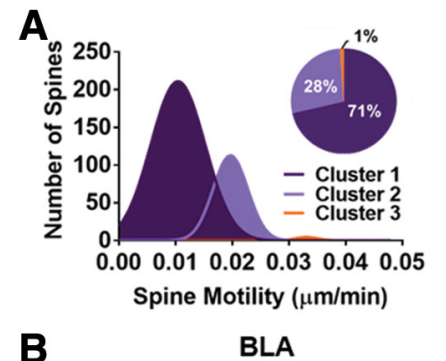

B
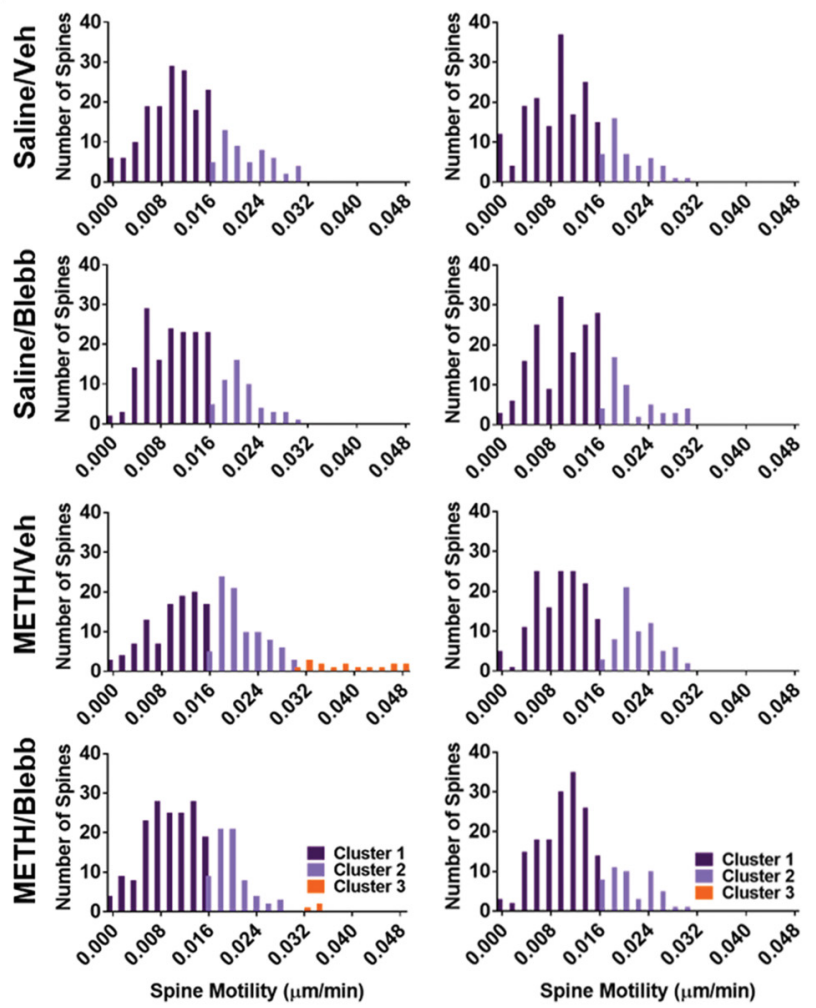

Figure 11. Cluster analysis of CA 1 and BLC spine movements following NMII inhibition. $A$, All CA1 and BLC spine movements were organized into three clusters based on cluster analysis. $\boldsymbol{B}$, Histograms plotting the number of spines in each cluster when the groups were graphed individually.
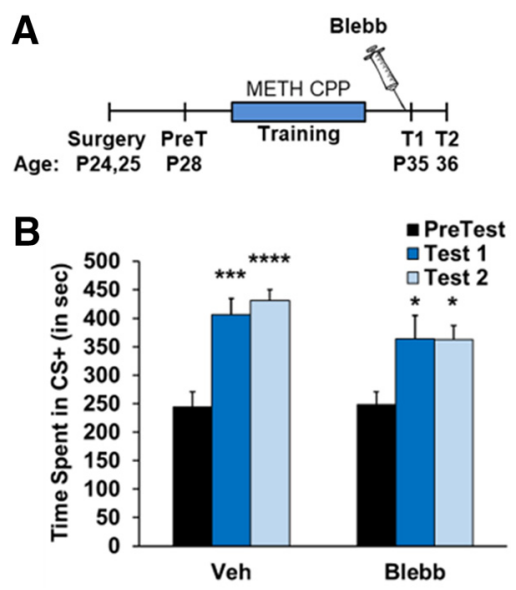

Figure 12. Intra-CA1 NMII inhibition has no immediate effect on an established METHassociated memory in adolescents. $\boldsymbol{A}$, Schematic of experimental design. $\boldsymbol{B}$, CPP data comparing time spent in the METH-paired CS + compartment before training (PreTest), $2 \mathrm{~d}$ after training, and 30 min after Blebb infusion (Test 1 ) and $3 \mathrm{~d}$ after training (no Blebb; Test 2; Veh, $n=10$; Blebb, $n=9$ ). Error bars represent SEM. ${ }^{*} p<0.05,{ }^{* * *} p<0.001$, **** $p<0.0001$. 
effect is that NMII is specifically involved in dynamic changes to the spine actin cytoskeleton and the remaining dynamics are driven by actin treadmilling that does not require NMII or by microtubule polymerization (Hu et al., 2008; Gu and Zheng, 2009; Jaworski et al., 2009). This would argue for the particular importance of more motile spines. Indeed, $\sim 75 \%$ of all BLA spines display low motility at P28-P35 $(<0.015 \mu \mathrm{m} / \mathrm{min})$ following saline training, with or without Blebb, and the remaining $25 \%$ display moderate motility $(0.015-0.03 \mu \mathrm{m} / \mathrm{min}$; Fig. $9 F)$. However, METH training shifts the distribution of BLA spines such that moderately and highly motile spines (clusters 2 and 3; $0.015-0.05 \mu \mathrm{m} / \mathrm{min}$ ) account for $50 \%$ of the total spines assessed. Remarkably, administering Blebb after METH training, but at least $24 \mathrm{~h}$ before slicing and imaging, results in a very precise return of spine motility distribution to control levels, not below (Fig. 9D, bottom right, cumulative distribution plot). The notion that NMII may not contribute to the low-level motility seen under control conditions is consistent with prior evidence from our group that NMII is activated by synaptic stimulation, rather than serving a housekeeping function (Rex et al., 2010; Ozkan et al., 2015). This is precisely what makes the sustained susceptibility of METH spines to Blebb of interest, as it suggests that NMII remains constitutively active days after METH training. The ability of NMII to drive actin polymerization is dependent upon two critical steps. First, the RLC of NMII must be phosphorylated to drive a conformation change that exposes the head of the heavy chain (MHC) of NMII, which contains the ATP binding site (Wendt et al., 2001). ATP provides the energy for the motor head of the MHC to physically slide actin filaments, driving cytoskeletal rearrangement (Vicente-Manzanares et al., 2009). Blebb works by interfering with the ATP binding site (Kovács et al., 2004). However, Blebb can influence NMII function only if the protein is activated via RLC phosphorylation. Thus, the ability of Blebb to disrupt actin-dependent spine motility days after the stimulation events of METH-associated learning suggests the RLC of NMII may need to remain phosphorylated. This could represent a point of divergence between METH-associated memory representation in the BLA versus CA1, as well as METH-associated memory versus memories for other conditioned stimuli, including footshock, food reward, and other drugs of abuse (Young et al., 2014, 2016; Briggs et al., 2017). Future studies will be directed at identifying the upstream signaling cascade regulating METH-induced NMII RLC phosphorylation, focusing on the possibility that METH recruits a unique set of players in the BLA that interferes with the normal postlearning mechanisms responsible for actin-myosin stabilization.

\section{References}

Araya R, Eisenthal KB, Yuste R (2006a) Dendritic spines linearize the summation of excitatory potentials. Proc Natl Acad Sci U S A 103:1879918804.

Araya R, Jiang J, Eisenthal KB, Yuste R (2006b) The spine neck filters membrane potentials. Proc Natl Acad Sci U S A 103:17961-17966.

Bonhoeffer T, Yuste R (2002) Spine motility: phenomenology, mechanisms, and function. Neuron 35:1019-1027.

Bouwmeester H, Smits K, Van Ree JM (2002) Neonatal development of projections to the basolateral amygdala from prefrontal and thalamic structures in rat. J Comp Neurol 450:241-255.

Briggs SB, Blouin AM, Young EJ, Rumbaugh G, Miller CA (2017) Memory disrupting effects of nonmuscle myosin II inhibition depend on the class of abused drug and brain region. Learn Mem 24:70-75.

Briggs SB, Hafenbreidel M, Young EJ, Rumbaugh G, Miller CA (2018) The role of nonmuscle myosin II in polydrug memories and memory reconsolidation. Learn Mem 25:391-398.
Brummelte S, Teuchert-Noodt G (2006) Postnatal development of dopamine innervation in the amygdala and the entorhinal cortex of the gerbil (Meriones unguiculatus). Brain Res 1125:9-16.

Childress AR, Mozley PD, McElgin W, Fitzgerald J, Reivich M, O'Brien CP (1999) Limbic activation during cue-induced cocaine craving. Am J Psychiatry 156:11-18.

Clement JP, Aceti M, Creson TK, Ozkan ED, Shi Y, Reish NJ, Almonte AG, Miller BH, Wiltgen BJ, Miller CA, Xu X, Rumbaugh G (2012) Pathogenic SYNGAP1 mutations impair cognitive development by disrupting maturation of dendritic spine synapses. Cell 151:709-723.

Dumitriu D, Cossart R, Huang J, Yuste R (2007) Correlation between axonal morphologies and synaptic input kinetics of interneurons from mouse visual cortex. Cereb Cortex 17:81-91.

Dunaevsky A, Tashiro A, Majewska A, Mason C, Yuste R (1999) Developmental regulation of spine motility in the mammalian central nervous system. Proc Natl Acad Sci U S A 96:13438-13443.

Fischer M, Kaech S, Knutti D, Matus A (1998) Rapid actin-based plasticity in dendritic spines. Neuron 20:847-854.

Fischer M, Kaech S, Wagner U, Brinkhaus H, Matus A (2000) Glutamate receptors regulate actin-based plasticity in dendritic spines. Nat Neurosci 3:887-894.

Gavin CF, Rubio MD, Young E, Miller C, Rumbaugh G (2012) Myosin II motor activity in the lateral amygdala is required for fear memory consolidation. Learn Mem 19:9-14.

Gu J, Zheng JQ (2009) Microtubules in dendritic spine development and plasticity. Open Neurosci J 3:128-133.

Hayashi-Takagi A, Yagishita S, Nakamura M, Shirai F, Wu YI, Loshbaugh AL, Kuhlman B, Hahn KM, Kasai H (2015) Labelling and optical erasure of synaptic memory traces in the motor cortex. Nature 525:333-338.

Hu X, Viesselmann C, Nam S, Merriam E, Dent EW (2008) Activitydependent dynamic microtubule invasion of dendritic spines. J Neurosci 28:13094-13105.

Jaworski J, Kapitein LC, Gouveia SM, Dortland BR, Wulf PS, Grigoriev I, Camera P, Spangler SA, Di Stefano P, Demmers J, Krugers H, Defilippi P, Akhmanova A, Hoogenraad CC (2009) Dynamic microtubules regulate dendritic spine morphology and synaptic plasticity. Neuron 61:85-100.

Kasai H, Matsuzaki M, Noguchi J, Yasumatsu N, Nakahara H (2003) Structure-stability-function relationships of dendritic spines. Trends Neurosci 26:360-368.

Kim CH, Lisman JE (1999) A role of actin filament in synaptic transmission and long-term potentiation. J Neurosci 19:4314-4324.

Koskinen M, Bertling E, Hotulainen R, Tanhuanpää K, Hotulainen P (2014) Myosin IIb controls actin dynamics underlying the dendritic spine maturation. Mol Cell Neurosci 61:56-64.

Kovács M, Tóth J, Hetényi C, Málnási-Csizmadia A, Sellers JR (2004) Mechanism of blebbistatin inhibition of myosin II. J Biol Chem 279:3555735563.

Kutlu MG, Gould TJ (2016) Effects of drugs of abuse on hippocampal plasticity and hippocampus-dependent learning and memory: contributions to development and maintenance of addiction. Learn Mem 23:515-533.

Lai CS, Franke TF, Gan WB (2012) Opposite effects of fear conditioning and extinction on dendritic spine remodelling. Nature 483:87-91.

Lamprecht R (2014) The actin cytoskeleton in memory formation. Prog Neurobiol 117:1-19.

Lendvai B, Stern EA, Chen B, Svoboda K (2000) Experience-dependent plasticity of dendritic spines in the developing rat barrel cortex in vivo. Nature 404:876-881.

Lordkipanidze T, Bikashvili T, Japaridze N, Zhvania M (2013) The effect of kainic acid on hippocampal dendritic spine motility at the early and late stages of brain development. Micron 49:28-32.

Luczynski P, Whelan SO, O’Sullivan C, Clarke G, Shanahan F, Dinan TG, Cryan JF (2016) Adult microbiota-deficient mice have distinct dendritic morphological changes: differential effects in the amygdala and hippocampus. Eur J Neurosci 44:2654-2666.

Majewska A, Sur M (2003) Motility of dendritic spines in visual cortex in vivo: changes during the critical period and effects of visual deprivation. Proc Natl Acad Sci U S A 100:16024-16029.

Makino H, Malinow R (2011) Compartmentalized versus global synaptic plasticity on dendrites controlled by experience. Neuron 72:1001-1011.

Mantzur L, Joels G, Lamprecht R (2009) Actin polymerization in lateral amygdala is essential for fear memory formation. Neurobiol Learn Mem 91:85-88. 
Matsuzaki M, Ellis-Davies GC, Nemoto T, Miyashita Y, Iino M, Kasai H (2001) Dendritic spine geometry is critical for AMPA receptor expression in hippocampal CA1 pyramidal neurons. Nat Neurosci 4:10861092.

Miller CA, Marshall JF (2005) Molecular substrates for retrieval and reconsolidation of cocaine-associated contextual memory. Neuron 47:873884.

Morris R (1985) Thy-1 in developing nervous tissue. Dev Neurosci 7: $133-160$.

Morton WM, Ayscough KR, McLaughlin PJ (2000) Latrunculin alters the actin-monomer subunit interface to prevent polymerization. Nat Cell Biol 2:376-378.

Noguchi J, Matsuzaki M, Ellis-Davies GC, Kasai H (2005) Spine-neck geometry determines NMDA receptor-dependent $\mathrm{Ca} 2+$ signaling in dendrites. Neuron 46:609-622.

Noguchi J, Nagaoka A, Watanabe S, Ellis-Davies GC, Kitamura K, Kano M, Matsuzaki M, Kasai H (2011) In vivo two-photon uncaging of glutamate revealing the structure-function relationships of dendritic spines in the neocortex of adult mice. J Physiol 589:2447-2457.

Omotade OF, Pollitt SL, Zheng JQ (2017) Actin-based growth cone motility and guidance. Mol Cell Neurosci 84:4-10.

Otis JM, Werner CT, Mueller D (2015) Noradrenergic regulation of fear and drug-associated memory reconsolidation. Neuropsychopharmacology 40:793-803.

Ozkan ED, Aceti M, Creson TK, Rojas CS, Hubbs CR, McGuire MN, Kakad PP, Miller CA, Rumbaugh G (2015) Input-specific regulation of hippocampal circuit maturation by non-muscle myosin IIB. J Neurochem 134:429-444.

Parnass Z, Tashiro A, Yuste R (2000) Analysis of spine morphological plasticity in developing hippocampal pyramidal neurons. Hippocampus 10:561-568.

Portera-Cailliau C, Pan DT, Yuste R (2003) Activity-regulated dynamic behavior of early dendritic protrusions: evidence for different types of dendritic filopodia. J Neurosci 23:7129-7142.

Rehberg K, Bergado-Acosta JR, Koch JC, Stork O (2010) Disruption of fear memory consolidation and reconsolidation by actin filament arrest in the basolateral amygdala. Neurobiol Learn Mem 94:117-126.

Rex CS, Gavin CF, Rubio MD, Kramar EA, Chen LY, Jia Y, Huganir RL, Muzyczka N, Gall CM, Miller CA, Lynch G, Rumbaugh G (2010) Myo- sin IIb regulates actin dynamics during synaptic plasticity and memory formation. Neuron 67:603-617.

Ryu J, Liu L, Wong TP, Wu DC, Burette A, Weinberg R, Wang YT, Sheng M (2006) A critical role for myosin IIb in dendritic spine morphology and synaptic function. Neuron 49:175-182.

Smart FM, Halpain S (2000) Regulation of dendritic spine stability. Hippocampus 10:542-554.

Star EN, Kwiatkowski DJ, Murthy VN (2002) Rapid turnover of actin in dendritic spines and its regulation by activity. Nat Neurosci 5:239-246.

Vicente-Manzanares M, Ma X, Adelstein RS, Horwitz AR (2009) Nonmuscle myosin II takes centre stage in cell adhesion and migration. Nat Rev Mol Cell Biol 10:778-790.

Wendt T, Taylor D, Trybus KM, Taylor K (2001) Three-dimensional image reconstruction of dephosphorylated smooth muscle heavy meromyosin reveals asymmetry in the interaction between myosin heads and placement of subfragment 2. Proc Natl Acad Sci U S A 98:4361-4366.

Xu T, Yu X, Perlik AJ, Tobin WF, Zweig JA, Tennant K, Jones T, Zuo Y (2009) Rapid formation and selective stabilization of synapses for enduring motor memories. Nature 462:915-919.

Yang G, Pan F, Gan WB (2009) Stably maintained dendritic spines are associated with lifelong memories. Nature 462:920-924.

Yarmola EG, Somasundaram T, Boring TA, Spector I, Bubb MR (2000) Actin-latrunculin A structure and function. differential modulation of actin-binding protein function by latrunculin A. J Biol Chem 275: $28120-28127$.

Young EJ, Aceti M, Griggs EM, Fuchs RA, Zigmond Z, Rumbaugh G, Miller CA (2014) Selective, retrieval-independent disruption of methamphetamineassociated memory by actin depolymerization. Biol Psychiatry 75:96-104.

Young EJ, Briggs SB, Miller CA (2015) The actin cytoskeleton as a therapeutic target for the prevention of relapse to methamphetamine use. CNS Neurol Disord Drug Targets 14:731-737.

Young EJ, Blouin AM, Briggs SB, Sillivan SE, Lin L, Cameron MD, Rumbaugh G, Miller CA (2016) Nonmuscle myosin IIB as a therapeutic target for the prevention of relapse to methamphetamine use. Mol Psychiatry 21: 615-623.

Young EJ, Briggs SB, Rumbaugh G, Miller CA (2017) Nonmuscle myosin II inhibition disrupts methamphetamine-associated memory in females and adolescents. Neurobiol Learn Mem 139:109-116. 